\title{
In Honour and Memory of Professor Tien H. Wu
}

\author{
Kok-Kwang Phoon ${ }^{1}$ and C. Hsein Juang ${ }^{2}$ \\ ${ }^{1}$ Dept. of Civil and Environmental Engineering, National University of Singapore, Singapore. \\ E-mail: kkphoon@nus.edu.sg \\ ${ }^{2}$ Dept. of Civil Engineering, National Central University, Taiwan. \\ E-mail: hsein@cc.ncu.edu.tw
}

Professor Emeritus Tien H. Wu passed away on June 7, 2018. A Georisk special issue (Vol. 13, Issue 4, 2019) was initiated by Georisk and ISSMGE TC304 to commemorate the passing of a founding member of the georisk community who has been instrumental in bringing probabilistic methods to geotechnical practice over a distinguished career of more than 50 years. He is fondly addressed as TH by many of his friends and colleagues. Although he was a humble person, he left a lasting impression on many members of our community who were fortunate enough to have interacted with him - this is apparent from the heartfelt words penned by the authors in a section "What do I remember about Professor Wu" at the end of their papers. Baecher and Christian (2019) observed that "TH brought enthusiasm and creativity to the emerging field of geotechnical reliability and mentored a generation of scholars and practitioners" in the lead paper of the special issue.

Some of the invited papers in this special issue will be presented in this Special Session in Honour and Memory of Professor Tien H. Wu in the 7th International Symposium on Geotechnical Safety and Risk (7th ISGSR), Taipei on 12 December 2019, between 2:40 - 4:10 PM and 4:30 - 6:00 PM at Room IB-101, International Building, National Taiwan University of Science and Technology (NTUST).

TH was a Professor Emeritus of The Ohio State University (he retired from OSU in 1994). His distinguished academic career includes 12 years of services as Assistant Professor, Associate Professor, and then Professor of Civil Engineering at Michigan State University, and 30 years of services as Professor of Civil Engineering at Ohio State University. For his life-long contributions to the profession of Civil Engineering, TH was conferred as an Honorary Member of ASCE in 2003. This is the highest honor one can receive from ASCE.

TH's research work covers a number of important subjects in geotechnical engineering, including strength properties of soil and rock, glaciology in Alaska and Antarctica, the stability of embankments and natural slopes, groundwater and seepage, soil-structure interaction of buried structures, risk and reliability assessment for foundations and slopes, and soil reinforcement. His work is of very high quality; among the many awards that TH has received is the ASCE State-of-the-Art Award in 1990 for his paper on "Reliability of Offshore Foundations."

There is little doubt that probabilistic approaches will be more widely applied in practice if more good examples, such as TH's case histories, are made available. An ISSMGE Global Survey on the State of the Art (SoA) and the State of the Practice (SoP) in geotechnical engineering was conducted between 10 March and 30 April 2017. ISSMGE TC304 (Engineering practice of risk assessment and management) posed the question: "What are the top three items in your 'wish list' that you feel would facilitate the adoption of reliability or riskinformed decision making in geotechnical practice?". More than half the respondents identified case studies and examples, soil/rock database, and statistical guidelines for site investigation data. As a result, TC304 launched a database sharing initiative called 304dB in 2018 (http://140.112.12.21/issmge/tc304.htm). TH would be gratified to see these developments. His research was recognized by the Ralph B. Peck Award from the Geo-Institute (2008), among other accolades. His strength and interest in documenting case histories and innovative solutions to geotechnical problems perhaps could be attributed to his early and continuous learning from Professor Peck. After all, TH was Professor Peck's first PhD from the University of Illinois at Urbana-Champaign (UIUC). Given his interest in drawing insights from case histories for decision making, it is not surprising that he is among the first to apply Bayesian methods in geotechnical engineering. Given the ever-increasing interest and use of Bayesian methods in geotechnical engineering in recent years, one may say that the geotechnical community has finally caught up with $\mathrm{TH}$.

To honour the memory of Professor Tien $\mathrm{H}$. Wu, we invited contributors to share case histories that demonstrate the practical value of probabilistic methods in geotechnical engineering. We believe his spirit will live on in this type of use-inspired research that he has tirelessly championed in his professional career. The special issue consists of 15 papers authored by some of the leading researchers in the field. The lead paper by Baecher and Christian (2019) entitled "TH Wu and the origins of geotechnical reliability" traced TH's seminal insights that shaped the early evolution of geotechnical reliability through the case histories he published. We are very pleased that some of the authors have graciously agreed to present their papers in this special session in honour and memory of Professor Tien H. Wu, which consists of 11 presentations. We invited Professor Daniel

Proceedings of the 7th International Symposium on Geotechnical Safety and Risk (ISGSR)

Editors: Jianye Ching, Dian-Qing Li and Jie Zhang

Copyright (c) ISGSR 2019 Editors. All rights reserved.

Published by Research Publishing, Singapore.

ISBN: 978-981-11-2725-0; doi:10.3850/978-981-11-2725-0_THWu-cd 
Pradel, who is a colleague of TH at Ohio State University, to share his reflections at the beginning of this session. Everyone is most welcome to attend.

Information on the T.H. Wu Fund is given at: https:/www.giveto.osu.edu/makeagift/?fund =647747

Special Session in Honour and Memory of Professor Tien H. Wu (Part 1)

2:40 - 4:10 PM, Room IB-101, International Building, NTUST

Chairs: Kok-Kwang Phoon and Charng Hsein Juang

In Honour and Memory of Professor Tien $\mathrm{H}$. Wu - opening remarks

Kok-Kwang Phoon and Charng Hsein Juang

T.H. Wu, a brilliant and unconventional pioneer

Daniel Pradel

Assessment of variability in soil properties from various field and laboratory tests

Ömer Bilgin, Kevin Arens, and Alexander Dettloff

A database of saturated hydraulic conductivity of fine-grained soils: probability density functions

Shuyin Feng and Paul J. Vardanega

Variation of CPTu- based transformation models for undrained shear strength of Finnish clays

Mika Knuuti and Tim Länsivaara

Probabilistic assignment of effective friction angles of sands and silty sands using quantile regression

Marco Uzielli and Paul W. Mayne

Uncertainty quantification of the bond stress - displacement relationship of shoring anchors in different geologic units

Rim Chahbaz, Salah Sadek, and Shadi Najjar

Special Session in Honour and Memory of Professor Tien H. Wu (Part 2)

4:30-6:00 PM, Room IB-101, International Building, NTUST

Chairs: Kok-Kwang Phoon and Charng Hsein Juang

Effect of extrapolation on interpreted capacity and model statistics of steel H-piles

Kok-Kwang Phoon and Chong Tang

On characteristic values and the reliability-based assessment of dykes

Michael A. Hicks, Divya Varkey, Abraham P. van den Eijnden, Tom de Gast, and Philip J. Vardon

Back analysis of settlement of Teven road trial embankment using Bayesian updating

Jinsong Huang, Cheng Zeng, and Richard Kelly

Probabilistic insights on a soil slope in San Francisco and a rock slope in Hong Kong

Bak Kong Low

Assessing effect of dynamic compaction on liquefaction potential using statistical methods - A case study Mengfen Shen, C. Hsein Juang, Chih-Sheng Ku, and Sara Khoshnevisan

Root-soil-water hydrological interaction and its impact on slope stability

Hong Zhu and Limin Zhang 